\section{Inactivating KISS1 Mutation and Hypogonadotropic Hypogonadism}

\author{
A. Kemal Topaloglu, M.D., Javier A. Tello, Ph.D., L. Damla Kotan, M.Sc., \\ Mehmet N. Ozbek, M.D., M. Bertan Yilmaz, Ph.D., Seref Erdogan, M.D., \\ Fatih Gurbuz, M.D., Fatih Temiz, M.D., Robert P. Millar, Ph.D., \\ and Bilgin Yuksel, M.D.
}

\section{SUMMARY}

Gonadotropin-releasing hormone $(\mathrm{GnRH})$ is the central regulator of gonadotropins, which stimulate gonadal function. Hypothalamic neurons that produce kisspeptin and neurokinin B stimulate GnRH release. Inactivating mutations in the genes encoding the human kisspeptin receptor (KISS1R, formerly called GPR54), neurokinin $\mathrm{B}$ (TAC3), and the neurokinin B receptor (TACR3) result in pubertal failure. However, human kisspeptin loss-of-function mutations have not been described, and contradictory findings have been reported in Kiss1-knockout mice. We describe an inactivating mutation in KISS1 in a large consanguineous family that results in failure of pubertal progression, indicating that functional kisspeptin is important for puberty and reproduction in humans. (Funded by the Scientific and Technological Research Council of Turkey [TÜBİTAK] and others.)

T IS STILL UNKNOWN HOW PUBERTY IN HUMANS, OCCURRING DURING THE early years of the second decade of life, is initiated. ${ }^{1}$ The hallmark of puberty is increased secretion of the gonadotropins, luteinizing hormone (LH) and folliclestimulating hormone (FSH), which act in concert to stimulate the gonads to drive sex-hormone secretion and gametogenesis. The production of gonadotropins from pituitary gonadotropic cells is controlled by the pulsatile delivery of GnRH. Inactivating mutations in the genes encoding GNRH1 ${ }^{2}$ or the GNRH receptor (GNRHR) ${ }^{3}$ give rise to normosmic idiopathic hypogonadotropic hypogonadism in humans. ${ }^{4}$ However, GnRH neurons lack sex-steroid receptors. This suggests the existence of GnRH-regulating neurons, which would mediate this effect.

A major breakthrough in identifying such candidate neurons was the finding that inactivating mutations in genes encoding the human kisspeptin receptor (KISS1R, formerly called GPR54), the cognate receptor for a hypothalamic peptide, kisspeptin, resulted in pubertal failure. ${ }^{4,5}$ More recently, mutations in TAC3 or TACR3 (encoding neurokinin $B$ and its receptor, respectively) were shown to result in the same phenotype. ${ }^{6}$ Kisspeptin and neurokinin $\mathrm{B}$ are coexpressed, along with dynorphin, in sex-hormone-responsive neurons in the arcuate nucleus (infundibular nucleus in primates), and their coordinated activity appears to regulate GnRH secretion. ${ }^{7}$ Gene defects associated with normosmic idiopathic hypogonadotropic hypogonadism have been described in all the neuropeptides and receptors identified as stimulators of GnRH except for the kisspeptin gene (KISS1).

Although Kiss1- and Kiss1r-knockout mouse models largely produce phenocopies (i.e., affected noncarriers) of human normosmic idiopathic hypogonadotropic hypogonadism resulting from inactivating mutations of KISS1R, there is evidence of remarkable residual activity of the hypothalamic-pituitary-gonadal axis. ${ }^{4,8-10}$ More-
From the Faculty of Medicine, Departments of Pediatric Endocrinology (A.K.T., F.G., F.T., B.Y.), Medical Biology (M.B.Y.), and Physiology (S.E.) and the Institute of Sciences, Department of Biotechnology (A.K.T., L.D.K.), Cukurova University, Adana; and Diyarbakir Children's Hospital, Department of Pediatric Endocrinology, Diyarbakir (M.N.O.) - both in Turkey; the University of Edinburgh, Centre for Integrative Physiology, Edinburgh (J.A.T., R.P.M.); and the University of Pretoria, Mammal Research Institute, Pretoria, and the University of Cape Town, Receptor Biology Group, Cape Town - both in South Africa (R.P.M.). Address reprint requests to Dr. Topaloglu at Cukurova University, Faculty of Medicine, Department of Pediatric Endocrinology, Adana 01330, Turkey, or at ktopaloglu@cu.edu.tr.

N Engl J Med 2012;366:629-35.

Copyright (c) 2012 Massachusetts Medical Society. 
over, a recent report challenged the requirement of kisspeptin signaling for pubertal maturation and fertility in female mice. ${ }^{11}$

Here, we describe a large consanguineous family in which normosmic idiopathic hypogonadotropic hypogonadism was associated with a lossof-function mutation of KISS1 in four affected sisters with pubertal failure. The findings indicate that kisspeptin is required for the initiation of puberty in humans.

\section{CASE REPORTS}

The proband (Patient II-8) had grown and developed normally until her early-to-mid-teen years. At 14.9 years of age, breast development was absent and the bone age was 13 years. Pelvic ultraso- nography revealed a hypoplastic uterus and ovaries lacking follicles. The proband's affected sisters (Patients II-1, II-7, and II-10) also had no spontaneous breast development. Clinical features including measures of growth, pubertal indexes, and circulating hormone concentrations in the proband and her affected sisters are shown in Table 1.

The pedigree of the family is shown in Figure 1A. All four affected sisters were otherwise healthy and had a normal sense of smell. The patients had normal anterior pituitary function other than the failure to undergo puberty. The parents were healthy paternal cousins of Kurdish origin. The mother underwent menarche at 12 years of age, and the father began having facial-hair growth at 14 years of age. The proband's unaffected, sexually mature sisters (II-2, II-4, II-6, and II-9) under-

\begin{tabular}{|c|c|c|c|c|c|}
\hline Characteristic & Patient II-1 & Patient II-7 & $\begin{array}{l}\text { Patient II-8 } \\
\text { (Proband) }\end{array}$ & Patient II-10 & $\begin{array}{l}\text { Normal Range } \\
\text { for Women } \\
\text { and Girls }\end{array}$ \\
\hline Age (yr) & 30 & 16 & 14 & 12 & \\
\hline Height (cm) & 168.1 & 166.1 & 152.1 & 140.0 & \\
\hline Body-mass indext & 20.6 & 19.4 & 17.4 & 18.5 & \\
\hline \multicolumn{6}{|l|}{ Tanner stage $\int$} \\
\hline Breast development & 5 & 1 & 1 & 1 & \\
\hline Pubic-hair development & 5 & 3 & 2 & 1 & \\
\hline $\mathrm{FSH}(\mathrm{mlU} / \mathrm{ml})$ & 1.7 & 0.7 & 0.2 & 0.4 & $2.5-10.2$ \\
\hline $\mathrm{LH}(\mathrm{mlU} / \mathrm{ml})$ & 0.1 & 0.2 & 0.0 & 0.2 & $1.9-12.5$ \\
\hline Estradiol (ng/dl) & 0.1 & 0.2 & 0.1 & 0.0 & $6.3-16.5$ \\
\hline \multicolumn{6}{|l|}{ GnRH stimulation test } \\
\hline Maximal FSH (mlU/ml) & NA & NA & 7.4 & NA & \\
\hline Maximal LH (mlU/ml) & NA & NA & 3.3 & NA & \\
\hline Prolactin $(\mathrm{pg} / \mathrm{ml})$ & NA & NA & 5.0 & NA & $2.8-29.2$ \\
\hline Thyrotropin (mlU/ml) & NA & 1.8 & 1.4 & 0.6 & $0.3-4.2$ \\
\hline Thyroxine (ng/dl) & NA & 1.0 & 1.1 & 1.1 & $0.9-1.8$ \\
\hline Cortisol ( $\mu \mathrm{g} / \mathrm{dl})$ & NA & 17.7 & 10.7 & NA & $3.0-25.0$ \\
\hline Dehydroepiandrosterone sulfate $(\mu \mathrm{g} / \mathrm{dl})$ & NA & NA & 77 & NA & $35-430$ \\
\hline Insulin-like growth factor 1 (ng/ml) & NA & NA & 271 & NA & $87-368$ \\
\hline \multicolumn{6}{|c|}{$\begin{array}{l}\text { - The coefficients of variation within and between hormonal assays were less than } 5 \% \text {. To convert the values for estradiol } \\
\text { to picomoles per liter, multiply by } 36.71 \text {. To convert the values for thyroxine to picomoles per liter, multiply by } 12.87 \text {. } \\
\text { To convert the values for cortisol to nanomoles per liter, multiply by } 27.59 \text {. To convert the values for dehydroepiandros- } \\
\text { terone sulfate to nanomoles per liter, multiply by } 27.21 \text {. FSH denotes follicle-stimulating hormone, GnRH gonadotropin- } \\
\text { releasing hormone, LH luteinizing hormone, and NA not available. } \\
\text { Patient II-1 had received estrogen-replacement therapy, resulting in Tanner stage } 5 \text { breast development. However, her } \\
\text { FSH, LH, and estradiol levels were measured while she was not receiving therapy. } \\
\text { The body-mass index is the weight in kilograms divided by the square of the height in meters. } \\
\text { The Tanner stage is used to describe breast and pubic-hair development, with } 1 \text { representing prepubertal status and } \\
5 \text { representing maturity. }\end{array}$} \\
\hline
\end{tabular}


went menarche around 12 years of age and had regular menses. The two adult siblings heterozygous for KISS1, a sister (II-2) and a brother (II-3), both had children without receiving any medical assistance. (For the complete case reports, see the Supplementary Appendix, available with the full text of this article at NEJM.org.)

\section{METHODS}

\section{STUDY CONDUCT}

The Mersin 1st Clinical Research Ethics Committee approved this study, and written informed consent was obtained from each adult participant and the parents of the participating children.

\section{LABORATORY STUDIES}

Plasma corticotropin and serum $\mathrm{FSH}$, estradiol, dehydroepiandrosterone sulfate, cortisol, and testosterone levels were analyzed with the use of commercial kits based on a solid-phase, two-site sequential or competitive chemiluminescent immunometric assay. Serum LH levels were determined with the use of a two-step immunometric sandwich assay and a luminometric technique

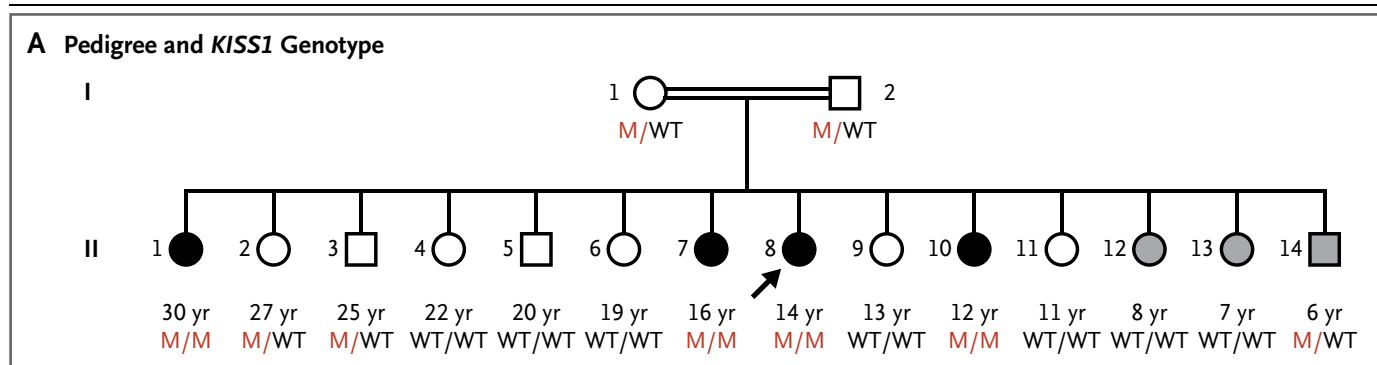

B Overnight Sleeping Profile

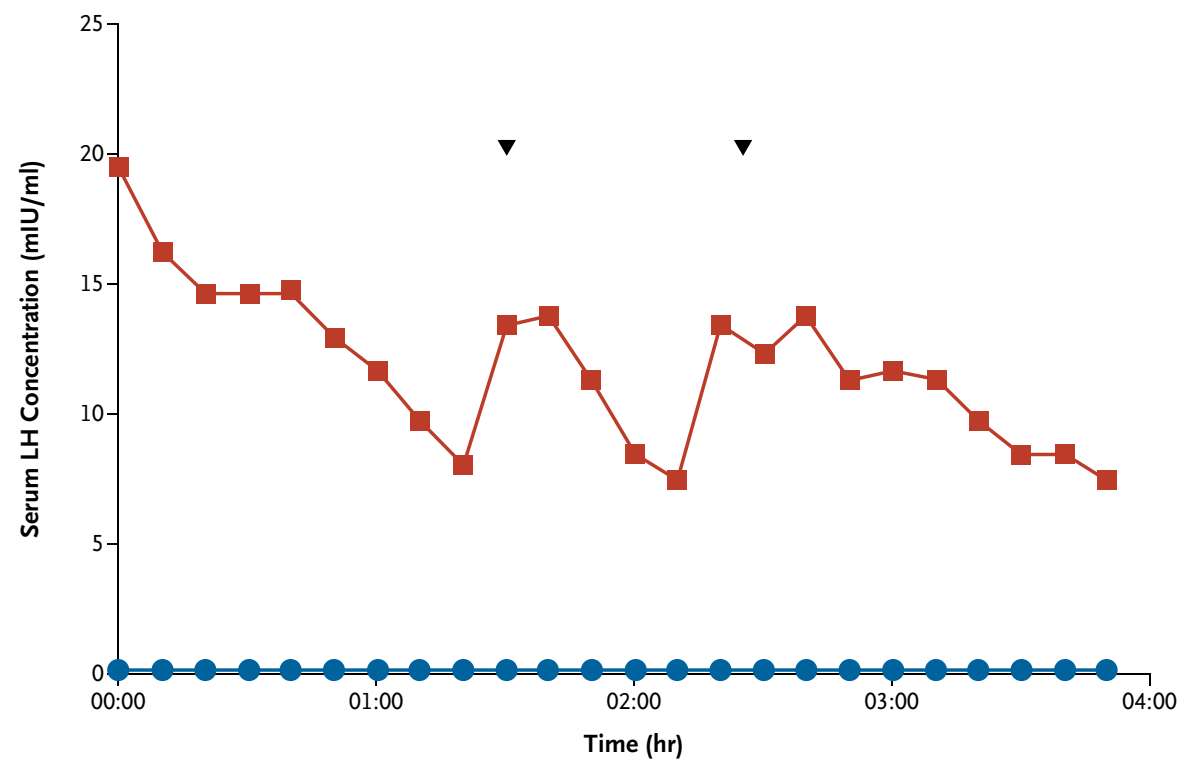

Figure 1. Pedigree and Luteinizing Hormone (LH) Profiles of the Proband's Family.

Panel A shows the pedigree of the proband (arrow) and her family. Solid circles indicate affected girls or women, open symbols unaffected family members, gray symbols family members of uncertain status (prepubertal), squares male family members, circles female family members, and the double line consanguinity. The age is shown under each symbol in generation II. Under every symbol is the KISS1 genotype, with M denoting mutant, and WT wild type. Panel B shows the overnight sleep LH profiles of the proband (Patient II-8, blue circles) and a healthy sister (Patient II-6, red squares), a KISS1 WT homozygote, in the midfollicular phase. The two arrowheads show the healthy sister's LH peaks. The proband's LH readings are all below the lower limit of detection of the assay, 0.2 mIU per milliliter. 
(Beckman Coulter). The intraassay and interassay coefficients of variation are less than $6 \%$ and less than $7 \%$, respectively. The lower limit of detection with a $95 \%$ confidence interval is $0.2 \mathrm{mIU}$ per milliliter.

We conducted a GnRH-stimulation test in the proband (Patient II-8) by injecting $0.1 \mathrm{mg}$ of GnRH intravenously and obtaining blood samples at 0 , 20,40 , and 60 minutes after injection for measurement of FSH and LH. Profiling of overnight LH levels during sleep was carried out during the midfollicular phase in the proband and a healthy sister (Patient II-6) who had a homozygous wildtype genotype for the KISS1 mutation (see below). Blood samples were obtained every 10 minutes for the 4 hours from midnight to 4 a.m. The pattern of LH secretion was analyzed according to a modified Santen and Bardin method. ${ }^{12}$

\section{GENOTYPING}

We performed automated sequencing of blood specimens from the proband for variants in known genes or strong candidate genes for idiopathic hypogonadotropic hypogonadism, including GNRHR, GNRH1, KISS1R, KISS1, the Kallmann syndrome 1 sequence gene (KAL1), the prokineticin 2 gene (PROK2) and PROK2 receptor gene (PROK2R), and the fibroblast growth factor receptor 1 gene (FGFR1). We performed genomewide analysis of single-nucleotide polymorphisms (SNPs) on microarrays (250K NspI SNP microarrays, Affymetrix) and analyzed the data using AutoSNPa software. ${ }^{13}$ This helps identify autozygous regions in affected persons, which are based on inheritance of the same ancestral mutant allele from both parents.

We also used whole-exome sequencing (selective sequencing of coding regions of the human genome) to identify the causative mutations. For exome sequencing, samples were prepared as an Illumina sequencing library, and in the second step, the sequencing libraries were enriched for the desired target according to the Illumina Exome Enrichment protocol. The captured libraries were sequenced (Illumina HiSeq 2000 Sequencer, Macrogen). The reads were mapped against the UC Santa Cruz Genome Browser (hg19 assembly). Identified mutations were checked in familial and sporadic cases of normosmic hypogonadotropic hypogonadism as well as in ethnically matched healthy adult controls in our study cohort.

\section{INOSITOL PHOSPHATE PRODUCTION}

Human kisspeptin-10 (amino acid sequence TyrAsn-Trp-Asn-Ser-Phe-Gly-Leu-Arg-Phe- $\mathrm{NH}_{2}$ ) was purchased from Sigma. Mutant human kisspeptin-10 (amino acid sequence Tyr-Asn-Trp-Lys-SerPhe-Gly-Leu-Arg-Phe- $\mathrm{NH}_{2}$ ) was custom-synthesized at a purity of at least $95 \%$ (EZBiolabs). Mutant and wild-type kisspeptin-10 were compared for their ability to stimulate inositol phosphate production in COS-7 cells transfected with KISS1R (see the Supplementary Appendix for details).

\section{RESULTS}

A GnRH stimulation test revealed a blunted response with maximum LH and FSH concentrations of 3.3 and $7.4 \mathrm{mIU}$ per milliliter, respectively. LH profiling during sleep (Fig. 1B) in the proband (Patient II-8) showed readings that were all below the lower limit of detection $(0.2 \mathrm{mIU}$ per milliliter). The LH readings in the healthy sister (Patient II-6), on the other hand, showed a normal adult female profile with two LH peaks.

Genomewide SNP analysis identified two regions of homozygosity common to all affected patients but not found in any unaffected family member. One of the regions, a $5.5-\mathrm{Mb}$ segment (from 201.6 Mb to 207.1 Mb) on chromosome 1, includes the KISS1 gene, which was a candidate for causing normosmic idiopathic hypogonadotropic hypogonadism.

We found homozygous nonsynonymous mutations in the coding sequence of KISS1 (Human Genome Organisation's Gene Nomenclature Committee [HGNC] number 6341) in all affected subjects (Fig. 2A). All affected family members were homozygous for a change from cytosine to guanine at complementary DNA nucleotide 345 (National Center for Biotechnology Informatics [NCBI] reference sequence NM_002256.3:c.345C $\rightarrow \mathrm{G}$ ), leading to the substitution of asparagine by lysine at residue 115 (NCBI reference sequence NP_002247.3:p.115 $\rightarrow \mathrm{K})$. This residue is conserved among all species with this gene (Fig. 2B). The Sorting Intolerant From Tolerant (SIFT) score for whether this amino acid substitution affects protein function is 0.00 , which corresponds to a very damaging effect (possible range, 0 to 1 , with scores $\leq 0.05$ predicting damage to protein function; http://sift.jcvi.org). This mutation was not found in 100 ethnically matched healthy adult 
Figure 2. Genetic Profile of the Proband's Family. Panel A shows the results of genotype sequencing of the kisspeptin-l gene (KISS1) in affected and unaffected members of the proband's family. Amino acids are labeled with the one-letter symbols at the top of the chromatograms, with the corresponding double-stranded nucleotide sequences below: the top line shows the homozygous mutant genotype, the middle line shows the heterozygous genotype, and the bottom line shows the homozygous wild-type genotype. We found a p.115N $\rightarrow \mathrm{K}$ mutation that resulted in a nucleotide change from $C$ to $\mathrm{G}$ (highlighted) at complementary DNA nucleotide 345 (National Center for Biotechnology Informatics [NCBI] reference sequence NM_002256.3:c.345C $\rightarrow \mathrm{G}$ ) and the replacement of asparagine by lysine at amino acid residue 115 (NCBI sequence NP_002247.3:p.115N $\rightarrow$ K). Panel $B$ shows that the mutated amino acid residue $\mathrm{N} 115$ is conserved among all species possessing orthologues or paralogues of KISS1. The reference sequence identifier is given in parentheses. Panel $\mathrm{C}$ shows the results of in vitro analysis of the functional consequence of the mutant kisspeptin-10 variant. Myo- $\left[2-{ }^{3} \mathrm{H}\right]$ inositol phosphate (IP) accumulation in COS-7 cells expressing human KISSI receptor (KISSIR) was induced with graded concentrations of kisspeptin-10 (KP-10) for 1 hour. The intracellular ${ }^{3} \mathrm{H}$-IP concentrations are shown as scintillation counts per minute (cpm). The I bars indicate means $( \pm \mathrm{SE})$ for triplicate samples from three or more independent experiments. KP-10 concentrations stimulating an IP response halfway between baseline and maximum $\left(\mathrm{EC}_{50}\right)$ were derived from the means and standard errors and were $\log _{10}$ transformed. The $P$ value was calculated with the use of Student's t-test.

controls (data not shown). No KISS1 mutations were found in 12 other families with more than 1 affected member or in 90 sporadic cases of normosmic idiopathic hypogonadotropic hypogonadism.

Analysis of whole-exome sequencing data, with particular attention to the autozygous regions identified in autozygosity mapping and other candidate genes, on the basis of studies in animals revealed that the only mutation to account for the phenotypes of the proband and her affected sisters is the KISS1 mutation.

Functional studies (Fig. 2C) showed that human KISS1R had significantly reduced sensitivity to the mutant kisspeptin-10 variant (i.e., the concentration leading to a response halfway between baseline and maximum $\left[\mathrm{EC}_{50}\right.$ ] value of $\left.3.7 \mu \mathrm{M}\right)$ as compared with wild-type kisspeptin-10 $\left(\mathrm{EC}_{50}\right.$ value of $1.8 \mathrm{nM})(\mathrm{P}<0.001)$. In addition, the mutant kisspeptin-10 variant was unable to stimulate a maximal inositol phosphate response at any of

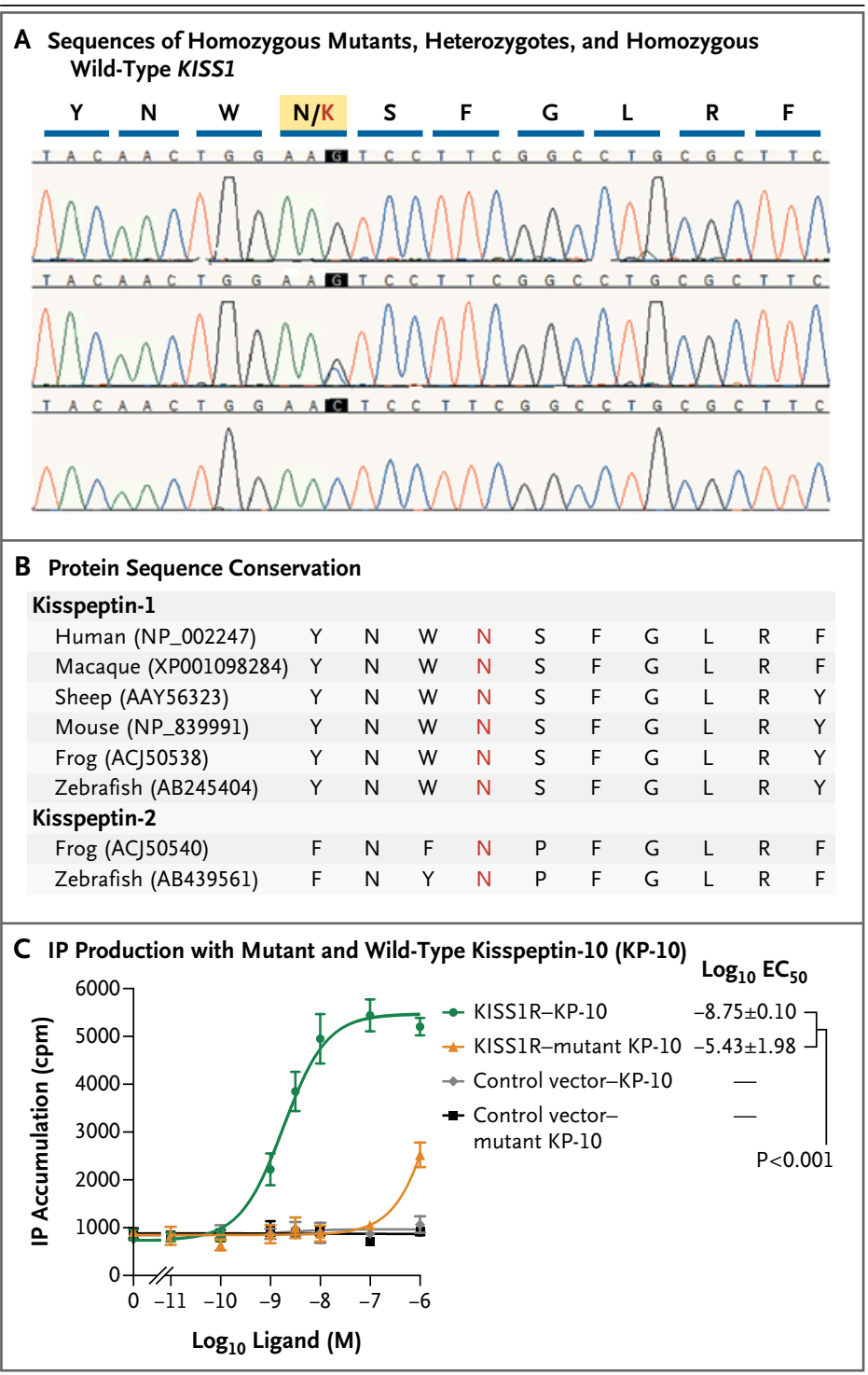

the concentrations tested, indicating significantly reduced efficacy as well as potency. Empty-vectortransfected COS-7 cells showed no inositol phosphate response to wild-type or mutant kisspeptin-10.

\section{DISCUSSION}

This large consanguineous family has four members with complete normosmic idiopathic hypogonadotropic hypogonadism, all of whom were found to have a KISS1 mutation. All affected family members were homozygous for the mutation, whereas their unaffected parents were heterozygous, and their unaffected siblings were either 
heterozygous or wild-type homozygous. The disorder was thus transmitted as an autosomal recessive trait, which indicates that one copy of KISS1 is sufficient for normal function of the gonadotropic axis, thus ruling out haploinsufficiency. Given the rarity of oligogenic inheritance in normosmic idiopathic hypogonadotropic hypogonadism, ${ }^{14}$ we ensured that the KISS1 mutation is the sole variant to account for the normosmic idiopathic hypogonadotropic hypogonadism phenotype in our patients. This was accomplished by combining a variety of methods, including candidate-gene screening, autozygosity mapping, and whole-exome sequencing.

In humans, kisspeptins derive from a common 145-amino acid precursor protein, which is proteolytically processed to peptides of 54,14 , 13, and 10 amino acids in length; all share the common carboxy-terminal decapeptide sequence YNWNSFGLRF- $\mathrm{NH}_{2}$, comprising kisspeptin-10, which is critical for biologic activity at the kisspeptin receptor. ${ }^{15,16}$ This decapeptide sequence is highly conserved during evolution (Fig. 2B). In addition, asparagine 4 (Asn4), which is mutated to lysine in our patients, is preserved in all known orthologues and paralogues.

Several groups have investigated the effects of structural modification of kisspeptin molecules. The five carboxy-terminal amino acids of kisspeptin-10, in particular residues $6,8,9$, and 10 , appear critical for agonist activity at the kisspeptin receptor. ${ }^{17-21}$ The next most important residue is Asn4; an alanine substitution for Asn4 in human kisspeptin-10 was approximately $25 \%$ as potent as the parent kisspeptin-10 in vivo. ${ }^{17} \mathrm{Nu}-$ clear magnetic resonance studies of kisspeptin have shown a helical structure spanning Asn 4 to phenylalanine 10; it appears that the helical structure plays an important role in receptor binding or activation. ${ }^{19}$ Thus, substitution of Asn 4 with lysine in our patients may disrupt this helical structure and account for the severely impaired ability to activate the kisspeptin receptor. Our data on in vitro receptor activation support this conclusion, since mutant kisspeptin was markedly less potent than the wild type. Studies of the development of antagonists have shown that substitutions for Asn 4 decrease binding affinity and may make contact with the receptor, ${ }^{20,22}$ both of which may contribute to the loss of activity of the mutant ligand.

Thus, the predicted consequence of the homo- zygous KISS1 mutation in the affected sisters in our study is a defective endogenous kisspeptin that is deficient in activating the kisspeptin receptor on the GnRH neurons and would result in the inability to elicit GnRH secretion - thereby accounting for the complete gonadotropin deficiency we have observed.

In the past 8 years, 26 patients with normosmic idiopathic hypogonadotropic hypogonadism have been reported to have loss-of-function mutations in KISS1R.23 Kiss1r- and Kiss1-knockout mouse models show a variable phenocopy of human KISS1R-inactivating mutations. A Kiss1-knockout mouse duplicated the human KISS1R mutant phenotype. ${ }^{8}$ However, a subgroup of another Kiss1knockout mouse model had a phenotype similar to the wild-type mouse. ${ }^{9}$ Chan and colleagues ${ }^{10}$ observed that nearly all Kiss1-knockout mice eventually underwent estrus. In general, the phenotype of the Kiss1-knockout model is less severe than that of the Kiss1r-knockout model. ${ }^{9}$ Likewise, a potent kisspeptin antagonist inhibits the rise in LH after castration in rodents and lowers LH-pulse amplitude after castration in sheep, but does not reduce basal LH concentrations; similarly, it inhibits GnRH pulsatility but does not lower the basal GnRH level in female pubertal monkeys. ${ }^{20,22}$ Taken together, these findings suggest that GnRH secretion may be more dependent on kisspeptin signaling in humans than in animal models.

Recently, Mayer and Boehm ${ }^{11}$ reported that ablation of KISS1-expressing cells in mice did not prevent progression of puberty or adult reproductive function. This observation contrasts with the impaired reproductive function reported in Kiss1knockout mice ${ }^{5,10-13}$ and the complete absence of reproductive function in our female patients with an inactivating KISS1 mutation. Given the stochastic nature of Cre recombinase expression during development in the Kiss1-Cre mice studied by Mayer and Boehm, it is possible that ablation of the Kiss1 neuron was incomplete, thus explaining the contrast in findings. Since only a small percentage of GnRH neurons is required for full reproductive function, ${ }^{24}$ the same explanation may apply to the inconsistency of findings regarding kisspeptin neurons.

In conclusion, we found that an inactivating mutation of KISS1 causes complete normosmic idiopathic hypogonadotropic hypogonadism in humans and would argue that kisspeptin signaling 
is a critical element in the human hypothalamicpituitary-gonadal axis.

Supported by the Scientific and Technological Research Council of Turkey (TÜBİTAK) (project number 109S455), the Interna- tional Centre for Genetic Engineering and Biotechnology (project number CRP/TUR 10-01), and the Cukurova University Scientific Research Projects Unit (project number BAP-26).

Disclosure forms provided by the authors are available with the full text of this article at NEJM.org.

\section{REFERENCES}

1. Plant TM. Hypothalamic control of the pituitary-gonadal axis in higher primates: key advances over the last two decades. J Neuroendocrinol 2008;20:719-26. 2. Bouligand J, Ghervan C, Tello JA, et al. Isolated familial hypogonadotropic hypogonadism and a GNRH1 mutation. $\mathrm{N}$ Engl J Med 2009;360:2742-8.

3. de Roux N, Young J, Misrahi M, et al. A family with hypogonadotropic hypogonadism and mutations in the gonadotropin-releasing hormone receptor. $\mathrm{N}$ Engl J Med 1997;337:1597-602.

4. Seminara SB, Messager S, Chatzidaki $\mathrm{EE}$, et al. The GPR54 gene as a regulator of puberty. N Engl J Med 2003;349:1614-27.

5. de Roux N, Genin E, Carel JC, Matsuda F, Chaussain JL, Milgrom E. Hypogonadotropic hypogonadism due to loss of function of the KiSS1-derived peptide receptor GPR54. Proc Natl Acad Sci U S A 2003;100:10972-6.

6. Topaloglu AK, Reimann F, Guclu M, et al. TAC3 and TACR 3 mutations in familial hypogonadotropic hypogonadism reveal a key role for neurokinin $B$ in the central control of reproduction. Nat Genet 2009;41:354-8.

7. Lehman MN, Coolen LM, Goodman RL. Minireview: kisspeptin/neurokinin B/ dynorphin (KNDy) cells of the arcuate nucleus: a central node in the control of gonadotropin-releasing hormone secretion. Endocrinology 2010;151:3479-89.

8. d'Anglemont de Tassigny X, Fagg LA, Dixon JP, et al. Hypogonadotropic hypogonadism in mice lacking a functional Kiss1 gene. Proc Natl Acad Sci U S A 2007, 104:10714-9.

9. Lapatto R, Pallais JC, Zhang D, et al. Kiss $1^{-1-}$ mice exhibit more variable hypo- gonadism than Gpr54--- mice. Endocrinology 2007;148:4927-36.

10. Chan YM, Broder-Fingert S, Wong KM, Seminara SB. Kisspeptin/Gpr54-independent gonadotrophin-releasing hormone activity in Kiss1 and Gpr54 mutant mice. J Neuroendocrinol 2009;21:1015-23. 11. Mayer C, Boehm U. Female reproductive maturation in the absence of kisspeptin/GPR54 signaling. Nat Neurosci 2011; 14:704-10.

12. Hayes FJ, McNicholl DJ, Schoenfeld D, Marsh EE, Hall JE. Free alpha-subunit is superior to luteinizing hormone as a marker of gonadotropin-releasing hormone despite desensitization at fast pulse frequencies. J Clin Endocrinol Metab 1999;84: 1028-36.

13. Carr IM, Flintoff KJ, Taylor GR, Markham AF, Bonthron DT. Interactive visual analysis of SNP data for rapid autozygosity mapping in consanguineous families. Hum Mutat 2006;27:1041-6.

14. Pitteloud N, Quinton R, Pearce S, et al. Digenic mutations account for variable phenotypes in idiopathic hypogonadotropic hypogonadism. J Clin Invest 2007; 117:457-63.

15. Kotani M, Detheux M, Vandenbogaerde $\mathrm{A}$, et al. The metastasis suppressor gene KiSS-1 encodes kisspeptins, the natural ligands of the orphan $\mathrm{G}$ protein-coupled receptor GPR54. J Biol Chem 2001; 276:34631-6.

16. Muir AI, Chamberlain L, Elshourbagy NA, et al. AXOR12, a novel human G protein-coupled receptor, activated by the peptide KiSS-1. J Biol Chem 2001;276:28969-75. 17. Curtis AE, Cooke JH, Baxter JE, et al. A kisspeptin-10 analog with greater in vivo bioactivity than kisspeptin-10. Am J
Physiol Endocrinol Metab 2010;298(2): E296-E303.

18. Orsini MJ, Klein MA, Beavers MP, Connolly PJ, Middleton SA, Mayo KH. Metastin (KiSS-1) mimetics identified from peptide structure-activity relationshipderived pharmacophores and directed small molecule database screening. J Med Chem 2007;50:462-71.

19. Gutiérrez-Pascual E, Leprince J, Martínez-Fuentes AJ, et al. In vivo and in vitro structure-activity relationships and structural conformation of Kisspeptin-10-related peptides. Mol Pharmacol 2009;76:5867.

20. Roseweir AK, Kauffman AS, Smith JT, et al. Discovery of potent kisspeptin antagonists delineate physiological mechanisms of gonadotropin regulation. J Neurosci 2009;29:3920-9.

21. Niida A, Wang Z, Tomita K, et al. Design and synthesis of downsized metastin (45-54) analogs with maintenance of high GPR54 agonistic activity. Bioorg Med Chem Lett 2006;16:134-7.

22. Millar RP, Roseweir AK, Tello JA, et al. Kisspeptin antagonists: unraveling the role of kisspeptin in reproductive physiology. Brain Res 2010;1364:81-9.

23. Wahab F, Quinton R, Seminara SB. The kisspeptin signaling pathway and its role in human isolated GnRH deficiency. Mol Cell Endocrinol 2011;346:29-36.

24. Silverman AJ, Zimmerman EA, Gibson MJ, et al. Implantation of normal fetal preoptic area into hypogonadal mutant mice: temporal relationships of the growth of gonadotropin-releasing hormone neurons and the development of the pituitary/testicular axis. Neuroscience 1985;16:69-84.

Copyright (c) 2012 Massachusetts Medical Society. in a public trials registry. The members of the International Committee of Medical Journal Editors (ICMJE) will consider most reports of clinical trials for publication only if the trials have been registered.

Current information on requirements and appropriate registries is available at www.icmje.org/faq_clinical.html. 